\title{
The role of vertical eddy flux in Southern Ocean heat uptake
}

\author{
A. K. Morrison, ${ }^{1}$ O. A. Saenko, ${ }^{2,3}$ A. McC. Hogg, ${ }^{1}$ and P. Spence ${ }^{3}$ \\ Received 25 August 2013; revised 5 October 2013; accepted 7 October 2013; published 17 October 2013.
}

[1] The role of changing vertical eddy heat flux on Southern Ocean heat uptake is investigated in an idealized eddy-permitting ocean model. Enhanced air-sea heat flux drives deep-reaching southern warming, due to a reduction in the isopycnal meridional temperature gradient and therefore decreased upward eddy heat flux. This mechanism is qualitatively similar in models with either permitted or parameterized eddies, due to its dependence on the isopycnal temperature gradient rather than the dynamical response of the eddy field. In contrast, increased wind stress drives mid-depth Southern Ocean cooling through an enhancement of the eddy field and resultant eddy heat divergence. The transient cooling extends over multiple decades while the mean flow adjusts to balance the faster eddy response. This wind-driven cooling mechanism has not been captured by coarse resolution models with fixed eddy parameterizations and is a possible candidate for the recent cooling observed in the Southern Ocean. Citation: Morrison, A. K., O. A. Saenko, A. McC. Hogg, and P. Spence (2013), The role of vertical eddy flux in Southern Ocean heat uptake, Geophys. Res. Lett., 40, 5445-5450, doi:10.1002/2013GL057706.

\section{Introduction}

[2] The oceans account for an overwhelming proportion ( $\sim 93 \%$ ) of the warming of the Earth system over the past 50 years [Levitus et al., 2012], and a large fraction of that ocean heat uptake has occurred in the upper $2000 \mathrm{~m}$ of the midlatitude Southern Ocean [Gille, 2008]. The intensified Southern Ocean warming has likely been driven by some component of the changing local surface forcing; a positive trend in the Southern Annular Mode has enhanced and shifted the westerly winds southward [Thompson and Solomon, 2002], surface air temperatures have increased [Chapman and Walsh, 2007] and evidence points toward increased precipitation over far southern latitudes due to an amplified hydrological cycle [Durack et al., 2012]. Yet the relative importance of the different forcing components and the mechanisms by which they may cause deep-reaching southern warming are unclear, due to a combination of limited

${ }^{1}$ ARC Centre of Excellence for Climate System Science and Research School of Earth Sciences, Australian National University, Canberra, ACT, Australia.

${ }^{2}$ Canadian Centre for Climate Modelling and Analysis, Environment Canada, Victoria, British Columbia, Canada.

${ }^{3}$ ARC Centre of Excellence for Climate System Science and Climate Change Research Centre, University of New South Wales, Sydney, New South Wales, Australia.

Corresponding author: A. K. Morrison, Research School of Earth Sciences, Australian National University, Canberra, ACT 0200, Australia. (adelem@princeton.edu)

(C)2013. American Geophysical Union. All Rights Reserved. 0094-8276/13/10.1002/2013GL057706 observations, uncertain atmospheric forcing, and insufficient modeling resolution.

[3] Meijers et al. [2011] divided the Southern Ocean temperature trend into an adiabatic component due to a wind-driven poleward migration of the Antarctic Circumpolar Current (as suggested by Gille [2008]) and a diabatic component due to changes in the vertical flux of heat from the surface and between ocean layers. The adiabatic component contributes a large warming trend, while the diabatic component contributes an opposing cooling trend, which acts to reduce the adiabatic trend by approximately half to produce the net observed deep warming. Meijers et al. [2011] do not attribute the cause of the diabatic cooling. Our aim in this paper is to investigate the dynamics of heat uptake processes which may be contributing to the diabatic trend in the Southern Ocean and in particular the role of eddies in modifying ocean temperature trends.

[4] The ocean heat content trend arising from diabatic processes will be reflected in changes in the time mean vertical heat flux budget. Numerical modeling studies have found that below the surface mixed layer, the globally averaged vertical flux of heat is primarily a balance between the winddriven mean circulation pumping heat downward and eddies (permitted or parameterized) transporting heat upward along isopycnals [Gregory, 2000; Wolfe et al., 2008]. It is reasonable to expect that eddies transport buoyancy (i.e., heat) upward in the global integral, because the baroclinic instability process acts to reduce available potential energy. Both the mean and eddy terms in the vertical heat flux budget are dominated by contributions from the Southern Ocean. Downward flux of heat by vertical diffusion in the tropics is only significant in the global budget in the upper few hundred meters of the ocean.

[5] Two main mechanisms for Southern Ocean heat uptake have been proposed - one driven by increased atmospheric surface temperature and the other by enhanced westerly winds. In the first mechanism, warming is amplified at mid-depths due to a reduced along-isopycnal meridional temperature gradient in a warmer climate and therefore decreased upward eddy heat flux, as shown in a coarse resolution model with parameterized isopycnal diffusion by Gregory [2000]. Huang et al. [2003] looked at the sensitivity of deep ocean heat uptake to a range of surface forcing and diffusivity perturbations and concluded that the large variation in deep ocean heat uptake between models arises primarily due to the high sensitivity to parameterized isopycnal diffusivity in the Southern Ocean and diapycnal diffusivity in the tropics. Brierley et al. [2008], in another coarse resolution coupled model study, found that reduced isopycnal diffusion was the largest contributor to the global imbalance leading to the warming trend between 350 and $1500 \mathrm{~m}$.

[6] Despite the dependence of this Southern Ocean warming mechanism on the eddy field, it has been largely untested 
in eddy-permitting or eddy-resolving models. Wolfe and Cessi [2009] investigated ocean heat uptake in an idealized eddy-resolving simulation with a northern hemisphere surface warming perturbation. In agreement with the coarse resolution study of Gregory [2000], the northern surface temperature gradient decreased in the warmer climate, reducing the vertical eddy heat flux and resulting in deep ocean heat uptake. However, in their model, density was a linear function of temperature only. With no isopycnal temperature gradient, the only mechanism available for eddies to alter the vertical heat budget is through a change in the potential energy that maintains the eddy activity. Whether the Southern Ocean vertical eddy heat flux decreases in response to surface warming due to a change in baroclinicity or due to a change in the isopycnal temperature gradient remains to be tested in an eddy-permitting or eddyresolving model.

[7] The second proposed mechanism for Southern Ocean heat uptake acts through a wind-driven enhancement of the downward mean heat flux and is the primary cause of Southern Ocean warming in the Third Coupled Model Intercomparison Project (CMIP3) 20th century experiments [Cai et al., 2010]. However, none of the CMIP3 models resolve eddies and are therefore missing the response of the eddy heat flux to changing wind stress [Hogg et al., 2008], which may significantly offset the wind-driven heat uptake. In this paper, we use a suite of wind stress and surface warming perturbations in an idealized eddy-permitting model to explore the role of the Southern Ocean eddy field on these two mechanisms of deep ocean heat uptake.

\section{Numerical Model}

[8] We use an idealized, $1 / 4^{\circ}$ eddy-permitting ocean sector configuration of the MITgcm [Marshall et al., 1997], with an experimental setup identical to that described in Hogg et al. [2013]. We refer the reader to Figure 1 of that paper for an illustration of the model bathymetry and surface forcing for the reference case. The model domain is a $40^{\circ}$ wide sector, extending from $70^{\circ} \mathrm{S}$ to $70^{\circ} \mathrm{N}$, with a southern reentrant channel containing a simple Drake Passage-like sill of $1800 \mathrm{~m}$ depth. Vertical resolution increases toward the surface over 36 vertical levels. The model is run in hydrostatic, Boussinesq mode, and no explicit eddy parameterization scheme is used. Horizontal viscosity/diffusion are minimized with a biharmonic lateral viscosity/diffusivity of $10^{11} \mathrm{~m}^{4} \mathrm{~s}^{-1}$. Background vertical diffusion is set to $10^{-5} \mathrm{~m}^{2} \mathrm{~s}^{-1}$, with a surface enhancement in the upper $300 \mathrm{~m}$ to generate appropriate mixed layer depths. The convective adjustment scheme also amplifies the background vertical diffusivity to remove unstable density profiles. A linear equation of state is employed, with both temperature and salinity dependence. The surface forcing is temporally invariant and zonally uniform, with prescribed wind stress, surface temperature relaxation and a fixed freshwater flux [see Hogg et al., 2013]. The model is close to equilibrium after a spin-up period of 2500 years, after which point perturbations are split off from the reference case and run for a further 100 years. The forcing undergoes a step change and is then kept steady in the new perturbed configuration for the entire 100 years. In the warming perturbations, the surface relaxation temperature is increased uniformly across the domain by $0.5^{\circ} \mathrm{C}, 1^{\circ} \mathrm{C}, 2^{\circ} \mathrm{C}$, or $4^{\circ} \mathrm{C}$ as specified in the analysis. For the wind stress perturbations, the prescribed wind stress south of $30^{\circ} \mathrm{S}$ is scaled by factors of $0.7,1.2$, 1.3 , and 2, as specified in the analysis. There is only minimal feedback between wind stress changes and local surface heat fluxes, meaning that we can easily separate the windand warming-induced changes.

\section{Mean Vertical Heat Flux}

[9] Vertical heat fluxes are calculated following Wolfe et al. [2008]. The time-mean heat budget is separated into the sum of the heat content tendency $(\mathcal{T})$, advective heat fluxes due to the mean flow $(\mathcal{M})$ and eddies $(\mathcal{E})$, and heat fluxes arising from parameterized subgrid scale processes $(\mathcal{P}$; including turbulent diffusion and convective adjustment) as follows:

$$
\mathcal{T}+\mathcal{M}+\mathcal{E}+\mathcal{P}=0 .
$$

If we integrate from the bottom $-H$ to a reference level $z_{o}$ and over a horizontal subdomain $\mathcal{A}$ (with boundary $\partial \mathcal{A}$ ), the first three terms are calculated as

$$
\begin{gathered}
\mathcal{T}=\rho_{o} c_{p} \int_{-H}^{z_{o}} \int_{\mathcal{A}} \frac{\Delta \theta}{\Delta t} \mathrm{~d} A \mathrm{~d} z \\
\mathcal{M}=\rho_{o} c_{p}\left(\int_{\mathcal{A}} \bar{w} \bar{\theta}\left(z_{o}\right) \mathrm{d} A+\int_{-H}^{z_{o}} \oint_{\partial \mathcal{A}} \bar{u}_{H} \bar{\theta} \mathrm{d} \sigma \mathrm{d} z\right) \\
\mathcal{E}=\rho_{o} c_{p}\left(\int_{\mathcal{A}} \overline{w^{\prime} \theta^{\prime}}\left(z_{o}\right) \mathrm{d} A+\int_{-H}^{z_{o}} \oint_{\partial \mathcal{A}} \overline{u_{H}^{\prime} \theta^{\prime}} \mathrm{d} \sigma \mathrm{d} z\right)
\end{gathered}
$$

where $c_{p}$ is the specific heat content, $\theta$ is potential temperature, $u_{H}$ is the horizontal component of velocity normal to the boundary, - is the temporal average over the time period $\Delta t$ and primed quantities indicate deviations from the temporal mean. $\mathrm{d} A$ is the differential area of the subdomain $\mathcal{A}$, while $\mathrm{d} \sigma$ is the differential length along its boundary $\partial \mathcal{A}$. The second term in equations (3) and (4) represents the heat flux through the lateral boundary of the subdomain. The subgrid scale term, $\mathcal{P}$, is not calculated explicitly, but rather as the residual of the first three terms in equation (1). The sign of $\mathcal{P}$ indicates whether convective or diffusive fluxes are dominating the subgrid scale term; in the polar regions, convection dominates and $\mathcal{P}$ is positive, indicating upward heat flux, while in the tropics, vertical diffusion dominates and $\mathcal{P}$ is negative, indicating downward heat flux.

[10] The vertical heat flux components for the reference case simulation, averaged over the entire domain, are shown in Figure 1a. Figures 1b-1d show the "vertical" heat flux (actually the net divergence, including the lateral terms in equations (3) and (4)) for three subdomains, divided according to the sign of the mean heat flux, following Wolfe and Cessi [2009]. At mid-depth ( 300-2000 m) and averaged over the entire domain, the primary balance is between downward heat flux by the mean flow and upward heat flux by eddies, both dominated by contributions from the Southern Ocean (Figure 1b) and consistent with the previous studies of Gregory [2000] and Wolfe et al. [2008]. The magnitude of the eddy heat flux is close to that in the eddyresolving MITgcm model analyzed in Wolfe et al. [2008]; the area-averaged mid-depth eddy flux in our eddy-permitting model is $20 \%$ less than in their higher resolution model. 


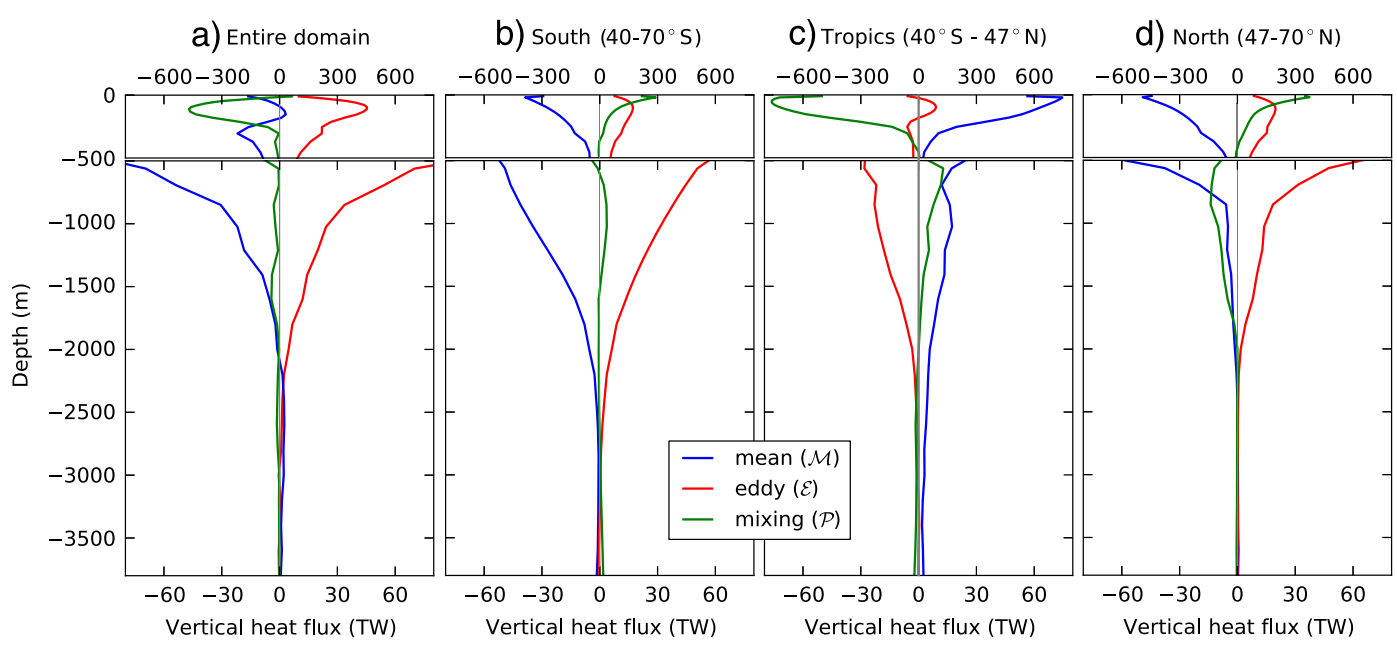

Figure 1. Vertical heat fluxes for the reference case, averaged over 10 years and different subdomains as follows: (a) the entire model domain, (b) the southern region $\left(40-70^{\circ} \mathrm{S}\right)$, (c) the tropics $\left(40^{\circ} \mathrm{S}-47^{\circ} \mathrm{N}\right)$, and (d) the northern region $\left(47-70^{\circ} \mathrm{N}\right)$. Note that the "vertical" mean and eddy fluxes, as defined in equations (3) and (4), represent the net divergence, including horizontal fluxes across the subdomain boundaries. Positive values indicate upward heat flux. Colors show the heat flux components as defined in section 3, with "mixing" being the sum of subgrid scale fluxes including parameterized convection and turbulent diffusion. The heat content tendency ( $\mathcal{T}$; not shown) is negligible. The horizontal scale has been increased below $500 \mathrm{~m}$ to show detail at depth.

In the upper ocean, where the model has increased vertical diffusivity (above $300 \mathrm{~m}$ ), the primary global balance is between downward diffusive heat flux from the tropics and upward heat flux by Southern Ocean eddies. Since we are primarily interested in the response of the vertical heat fluxes to forcing changes, we refer the reader to Wolfe et al. [2008] for a more in-depth explanation and regional analysis of the time mean vertical heat flux.

\section{Response to Idealized Climate Change Scenarios}

\subsection{Surface Warming}

[11] We first consider the response to a uniform $+2^{\circ} \mathrm{C}$ surface warming perturbation. The ocean warms (i.e., $\mathcal{T}>0$ ) due to changes in each of the mean, eddy, and subgrid scale vertical heat fluxes (solid lines in Figure 2a). The large warming in the upper $300 \mathrm{~m}$ is concentrated in the tropics (not shown) and arises from both increased downward diffusive heat flux and decreased upward mean heat flux. At mid-depths $(\sim 300-2000 \mathrm{~m})$, a significant fraction of the heat uptake occurs in the southern region (dashed lines in Figure 2a) and is predominantly due to a decrease in the upward eddy heat flux, consistent with the atmospheric warming driven mechanism identified in previous coarse resolution studies with parameterized eddies [Gregory, 2000; Huang et al., 2003; Brierley et al., 2008]. As time progresses over the 100 years of the warming perturbation, the anomalous vertical eddy heat flux remains the dominant cause of heat uptake in the global integral in the depth range 300-1000 $\mathrm{m}$ and the dominant cause of heat uptake in the southern region down to $2000 \mathrm{~m}$. Below $1000 \mathrm{~m}$ in the global integral, the change in the mean vertical heat flux becomes increasingly significant in the heat uptake over the first century. The response is qualitatively similar for warming perturbations of different magnitudes.
[12] The upward eddy heat flux in the mid-depth southern region scales inversely with the magnitude of the surface warming perturbation (Figure 2b). In the Southern Ocean, eddies flux heat along tilted isopycnals and the vertical eddy heat flux shown in Figure $2 \mathrm{a}$ is actually the vertical component of the along-isopycnal heat flux. Strong precipitation over the Southern Ocean leads to surface waters that are fresher than deep waters. Considered on an isopycnal, this salinity gradient necessitates a negative along-isopycnal temperature gradient (i.e., colder surface waters), since these quantities must compensate, to first order, on an isopycnal. Assuming downgradient, along-isopycnal tracer diffusion [e.g., Redi, 1982] of the form $\overline{v^{\prime} \theta^{\prime}} \rho=-\kappa \bar{\theta}_{y} \rho$ (where $(\cdot)^{\rho}$ indicates we are using an isopycnal framework), it is possible to trace the cause of the reduced upward eddy heat flux and hence the mid-depth warming in the southern region to the change in the along-isopycnal meridional temperature gradient (Figure 2c). In agreement with the coarse resolution study of Gregory [2000], the upward eddy heat flux in the southern region decreases due to faster warming in the surface region than at depth and a consequent reduction in the rate of along-isopycnal eddy mixing of temperature. The isopycnal temperature gradient across southern latitudes decreases as the surface relaxation temperature is increased, scaling similarly to the vertical eddy heat flux (Figure $2 b$ ).

\subsection{Enhanced Southern Wind Stress}

[13] Previous Southern Ocean observational and modeling studies have found a fast (2-3 years) and approximately linear response of the eddy kinetic energy to increasing wind stress [e.g., Meredith and Hogg, 2006; Hogg et al., 2008]. The enhancement of the eddy field with wind stress is a release of available potential energy, leading to an increase in the upward vertical eddy heat flux in the southern region (Figure 3a). This is the opposite response compared with the surface warming perturbation described in section 4.1 (Figure 2a). The mean vertical downward heat 

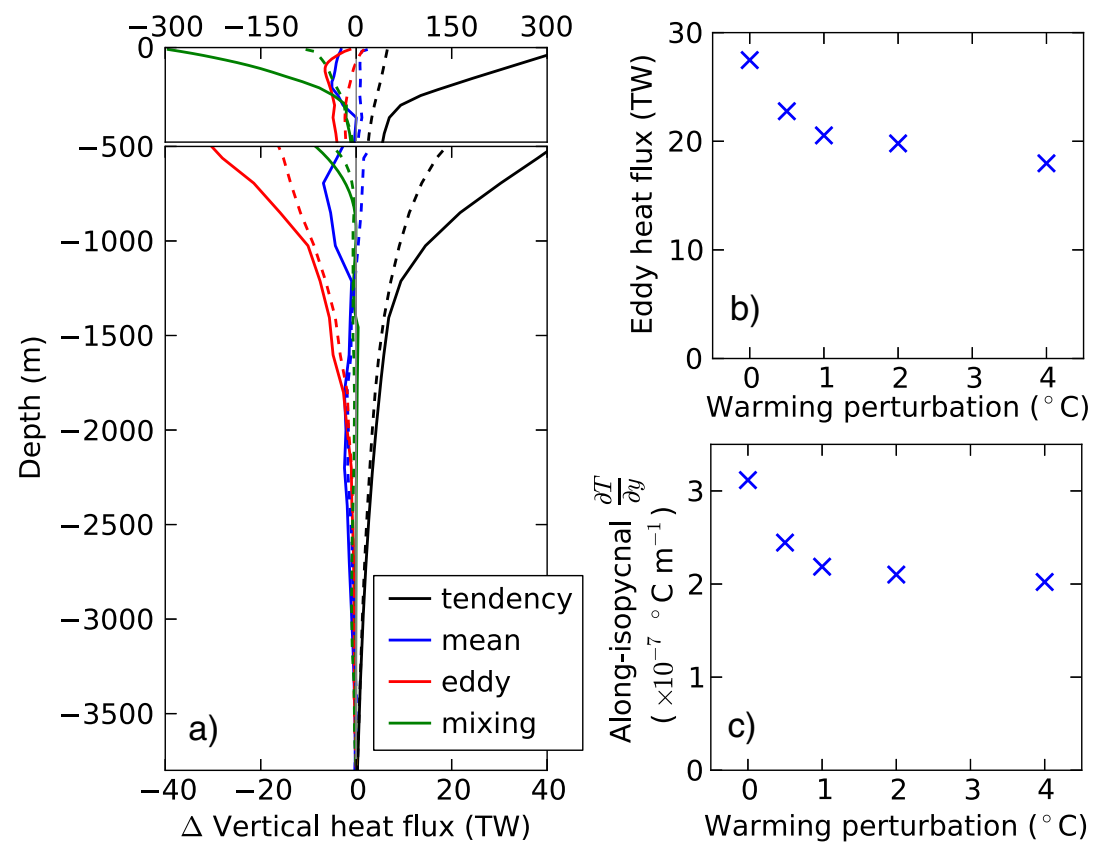

Figure 2. Vertical heat flux response to a uniform $+2{ }^{\circ} \mathrm{C}$ warming perturbation. (a) Change from the equilibrium vertical heat fluxes, averaged over the first 10 years of the perturbation, integrated over the entire domain (solid lines) and the contribution from the southern region (dashed lines). Heat flux components (colors) are defined as in Figure 1, with the additional heat content tendency, $\mathcal{T}$ (black). (b) Scaling of the vertical eddy heat flux in the southern region (i.e., the blue line in Figure 1b), averaged over the depth range 700-1600 m. (c) Scaling of the along-isopycnal, meridional temperature gradient on a mid-depth isopycnal $\left(1500 \mathrm{~m}\right.$ depth at $42^{\circ} \mathrm{S}, 700 \mathrm{~m}$ depth at $\left.62^{\circ} \mathrm{S}\right)$.
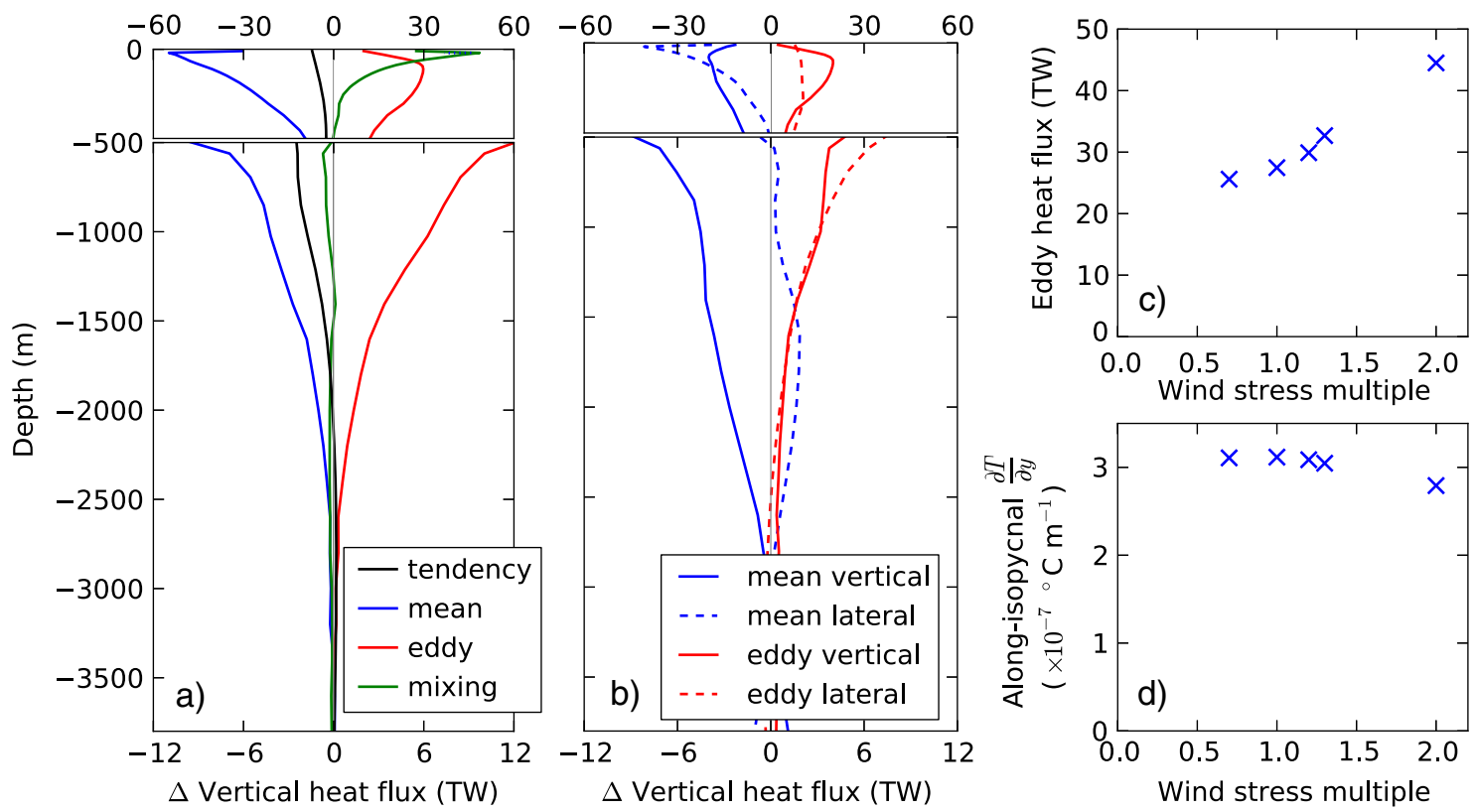

Figure 3. Heat flux response in the southern region $\left(40-70^{\circ} \mathrm{S}\right.$ only) to a $30 \%$ increase in Southern Ocean wind stress. (a) Change from the equilibrium vertical heat fluxes (net heat divergence) in the southern region, averaged over the first 10 years of the perturbation. Heat flux components (colors) are defined as in Figure 1, with the additional heat content tendency, $\mathcal{T}$ (black). (b) Separation of the net mean and eddy heat fluxes shown in Figure 3a into true vertical (solid lines) and lateral (dashed lines) components. (c) Scaling of the net eddy heat flux in the southern region, averaged over the depth range 700$1600 \mathrm{~m}$. (d) Scaling of the along-isopycnal, meridional temperature gradient on a mid-depth isopycnal (1500 $\mathrm{m}$ depth at $42^{\circ} \mathrm{S}, 700 \mathrm{~m}$ depth at $62^{\circ} \mathrm{S}$ ). 
flux associated with the upper overturning cell acts to deepen isopycnals on the northern side of the circumpolar current and thereby to warm at mid-depth; the timescale for this process is advective and therefore slower than the eddy response. Although we refer to the "vertical" mean and eddy heat fluxes in the southern region, these include significant contributions from lateral fluxes across the subdomain boundary at $40^{\circ} \mathrm{S}$. Figure $3 \mathrm{~b}$ shows the separation of the net heat divergence into the true vertical and lateral components.

[14] The net result of the mean and eddy responses is a small transient cooling trend in the mid-depth levels of the southern region. The cooling continues for around 60 years, until the mean flow increases sufficiently to balance the change in the eddy flux. Despite minimal change in the local surface heat flux, cooling is able to extend throughout the water column due to changes in the lateral components of the mean and eddy heat fluxes (shown in Figure 3b). Analysis of a range of wind stress perturbations indicates a near oneto-one relationship between the mid-depth eddy heat flux and the prescribed wind stress (Figure 3c). In contrast to the surface warming perturbations, the along-isopycnal temperature gradient remains approximately constant for small $(\sim \pm 30 \%)$ wind stress perturbations (Figure $3 \mathrm{~d}$ ). The change in eddy heat flux is instead driven by increased baroclinicity (i.e., the eddy heat flux scales with the eddy kinetic energy). Although the mid-depth southern region cools following the change in wind forcing, the net heat uptake integrated over the entire domain is positive (not shown), due to a reduction in the mean upward heat flux in the tropics.

\subsection{Combined Warming and Enhanced Wind Stress}

[15] The response of the vertical eddy heat flux to a wind stress perturbation (Figure 3c) has the opposite sign compared with the response to a warming perturbation (Figure 2b). If the two perturbation responses were to combine in an approximately linear way, the increase in westerly winds could partially reduce surface heat flux-driven warming in the mid-depth Southern Ocean. To explore this possibility, we have run a combined surface warming (uniform $\left.+2^{\circ} \mathrm{C}\right)$ and enhanced southern wind stress $\left(1.3 \times \tau_{o}\right)$ perturbation experiment. As would be expected from the linear sum of the separate warming and wind stress experiments, the net heat uptake integrated over the entire domain is larger in the combined perturbation than in either of the independent perturbations. However, in the southern region, the addition of the enhanced wind stress to the warming perturbation results in a small decrease in the heat uptake, compared with the surface warming only perturbation (not shown); this mechanism may account for the observed recent cooling in the mid-depth Southern Ocean that was inferred to be due to diabatic processes [Meijers et al., 2011]. The southern region heat uptake is reduced for a period of $\sim 60$ years, while the mean flow adjusts to the increased wind stress. After the mean heat flux has adjusted to balance the change in the eddy heat flux, the rate of heat uptake matches that in the warming only perturbation. The cumulative heat uptake in the southern region, integrated over full depth, is reduced by $5 \%$ over the 100 years of simulation.

\section{Discussion and Conclusions}

[16] We have used an eddy-permitting numerical model to investigate two mechanisms of mid-depth Southern Ocean temperature change, both of which rely on changes in the vertical eddy heat flux. The first mechanism, driven by increased surface heat flux and identified in a coarse resolution model by Gregory [2000], causes mid-depth warming through a reduction in the upward eddy heat flux. In a warmer climate, the along-isopycnal meridional temperature gradient decreases, leading to reduced isopycnal diffusion of temperature. Since the mechanism does not depend on a change in the eddy field, the response of the vertical eddy heat flux in our eddy-permitting model is qualitatively similar to that found in coarse resolution simulations with parameterized isopycnal diffusion.

[17] The second mechanism involves a response to increased wind stress forcing, which causes a transient cooling in the mid-depth southern region due to the faster response of the vertical eddy heat flux compared with the vertical mean heat flux. The upward eddy heat flux scales with the eddy kinetic energy and therefore responds on a timescale of $2-3$ years, while the downward mean heat flux changes on the timescale for the adjustment of the upper overturning circulation, which in our model is $\sim 60$ years. During this adjustment, a transient cooling occurs in the middepth southern region. Note that the relation between the mean and eddy components in the Southern Ocean vertical heat budget is quite different to that in the overturning circulation; at equilibrium, the vertical eddy heat flux nearly exactly opposes the vertical mean heat flux (Figure 1b), while the mean component of the overturning is significantly larger than the eddy component of the overturning [Morrison and Hogg, 2013]. Unlike in the surface warming mechanism, the response of the vertical eddy heat flux to enhanced wind stress may differ greatly between coarse resolution and eddy-permitting or eddy-resolving models. Indeed, in the CMIP3 models, enhanced wind stress drives Southern Ocean warming, rather than cooling, due to the unresponsive eddy parameterizations in these coarse resolution models [Cai et al., 2010].

[18] Acknowledgments. A.M.H. was supported by Australian Research Council Future Fellowship FT120100842. The numerical model was run on the NCI National Facility in Canberra, Australia, which is supported by the Australian Commonwealth Government.

[19] The Editor thanks two anonymous reviewers for their assistance in evaluating this paper.

\section{References}

Brierley, C. M., M. Collins, and A. J. Thorpe (2008), The impact of perturbations to ocean-model parameters on climate and climate change in a coupled model, Clim. Dyn., 34, 325-343.

Cai, W., T. Cowan, S. Godfrey, and S. Wijffels (2010), Simulations of processes associated with the fast warming rate of the southern midlatitude ocean, J. Clim., 23, 197-206.

Chapman, W. L., and J. E. Walsh (2007), A synthesis of Antarctic temperatures, J. Clim., 20, 4096-4117.

Durack, P. J., S. E. Wijffels, and R. J. Matear (2012), Ocean salinities reveal strong global water cycle intensification during 1950 to 2000 , Science, $336,455-458$.

Gille, S. T. (2008), Decadal-scale temperature trends in the Southern Hemisphere ocean, J. Clim., 21, 4749-4765.

Gregory, J. M. (2000), Vertical heat transports in the ocean and their effect on time-dependent climate change, Clim. Dyn., 16, 501-515.

Hogg, A. McC., M. P. Meredith, J. R. Blundell, and C. Wilson (2008), Eddy heat flux in the Southern Ocean: Response to variable wind forcing, J. Clim., 21, 608-620.

Hogg, A. McC., H. A. Dijkstra, and J. A. Saenz (2013), The energetics of a collapsing meridional overturning circulation, J. Phys. Oceanogr., 43, 1512-1524, doi:10.1175/JPO-D-12-0212.1.

Huang, B., P. H. Stone, and C. Hill (2003), Sensitivities of deep-ocean heat uptake and heat content to surface fluxes and subgrid-scale parameters in 
an ocean general circulation model with idealized geometry, J. Geophys. Res., 108, 3015, doi:10.1029/2001JC001218.

Levitus, S., et al. (2012), World ocean heat content and thermosteric sea level change (0-2000 m), 1955-2010, Geophys. Res. Lett., 39, L10603, doi:10.1029/2012GL051106.

Marshall, J., A. Adcroft, C. Hill, L. Perelman, and C. Heisey (1997), A finite-volume, incompressible Navier Stokes model for studies of the ocean on parallel computers, J. Geophys. Res., 102, 5753-5766.

Meijers, A. J. S., N. L. Bindoff, and S. R. Rintoul (2011), Frontal movements and property fluxes: Contributions to heat and freshwater trends in the Southern Ocean, J. Geophys. Res., 116, C08024, doi:10.1029/ 2010JC006832.

Meredith, M. P., and A. McC. Hogg (2006), Circumpolar response of Southern Ocean eddy activity to a change in the Southern Annular Mode, Geophys. Res. Lett., 33, L16608, doi:10.1029/2006GL026499.
Morrison, A. K., and A. McC. Hogg (2013), On the relationship between Southern Ocean Overturning and ACC transport, J. Phys. Oceanogr., 43, $140-148$.

Redi, M. H. (1982), Oceanic isopycnal mixing by coordinate rotation, J. Phys. Oceanogr., 12, 1154-1158.

Thompson, D. W. J., and S. Solomon (2002), Interpretation of recent Southern Hemisphere climate change, Science, 296, 895-899.

Wolfe, C. L., and P. Cessi (2009), Overturning circulation in an eddyresolving model: The effect of the pole-to-pole temperature gradient, J. Phys. Oceanogr., 39, 125-142.

Wolfe, C. L., P. Cessi, J. L. McClean, and M. E. Maltrud (2008), Vertical heat transport in eddying ocean models, Geophys. Res. Lett., 35, L23605, doi:10.1029/2008GL036138. 